\title{
Sternoclavicular Joint Transfer in the Management of Temporomandibular Joint Ankylosis- A case report
}

\author{
Md. Asaduzzaman ${ }^{1}$ Shohda Khatun² AHM Zahurul Huq ${ }^{3}$
}

\begin{abstract}
Temporomandibular joint ankylosis is a clinical condition resulting in loss of jaw function and retardation of growth. The main objectives of the treatment for Temporomandibular joint ankylosis are to increase mouth opening, restore joint function, initiation of mandibular growth, improve the patient's facial esthetic profile and prevent reankylosis. To release of ankylotic mass and gap is filled up with interpositional material. We reported a case of temporomandibular joint ankylosis in a 15 years old girl, whose treatment was done in the Department of Oral and Maxillofacial Surgery,Bangabondhu Sheikh Mujib Medical University, with sternoclavicular joint transfer which is very rare in our country and this was nearly fulfill treatment objective.
\end{abstract}

Keywords: Ankylosis, Temporomandibular joint(TMJ), sternoclavicular joint (SCJ);

Assistant professor, Department of Oral and Maxillofacial Surgery, Bangabondhu Sheikh Mujib Medical University, Shahbag, Dhaka

${ }^{2}$ Associate professor, Department of Oral and Maxillofacial Surgery, Bangabondhu Sheikh Mujib Medical University, Shahbag, Dhaka

${ }^{3}$ Professor, Department of Otolaryngology-Head \& Neck Surgery, Bangabandhu Sheikh Mujib Medical University, Shahbag, Dhaka

\section{Address ofcorrespondence:}

Dr. Md. Asaduzzaman, Assistant professor,

Department of Oral and Maxillofacial Surgery,

Bangabondhu Sheikh Mujib Medical University,

Shahbag, Dhaka.

Phone: 01912574985, Gmail: asaduzzamanuzzal.bsmmu@gmail.com.

\section{Introduction}

Temporomandibular joint ankylosis is one of the most distressing clinical conditions resulting in loss of jaw function and impairment of growth. The Temporomandibular joint ankylosis leads to facial asymmetry resulting in psychosocial problems in the affected patients. Unilateral ankylosis is associated with chin deviation to the affected side, vertical deficiency of the maxilla and the mandible on the side of defect, retrognathic mandible with short ramus, convex facial profile, absent or deficient cervico-mental angle, fullness of face on affected side, flatteneing on unaffected side, and prominent antigonial notch. The intra oral features includes occlusal cant with deviation of maxillary and mandibular midlines toward the affected side, class II malocclusion although class I occlusion may occasionally be seen, posterior cross bite, severe oral hygiene problems leading to caries and dental problems. Treatment for Temporomandibular joint ankylosis are to improve mouth opening, restore joint function, allow for mandibular growth, improve the patient's facial profile and prevent reankylosis. ${ }^{1}$ The etiology of recurrence of ankylosis has been postulated to be failure to have aggressive physiotherapy, poor compliance by the patient. Kaban postulated that recurrence could be due to inadequate excision of ankylotic mass ${ }^{2}$ In 
view of the technical difficulties and high incidence of recurrence the surgical management of temopormandibular joint ankylosis poses a significant challenge to both the surgeons and the patients. The various techniques for surgical correction of TMJ ankylosis are gap arthroplasty, interpositional arthroplasty, and joint reconstruction. ${ }^{3}$

Numerous materials have been suggested for reconstructing of TMJ. Traditionally, the costochondral grafts have been used for reconstruction of TM Joint in ankylosis patients, but because of the unpredictable growth, warpage, and fracture of the costochondral grafts have created a background for dissatisfaction and prompted a search for an alternative. ${ }^{4}$ It is observed that in humans the sternoclavicular joint and temporomandibular joint are similar anatomically and physiologically. ${ }^{5}$ This similariy of the joints have encouraged the surgeons to use SCG as an alternative to costochondral grafts. The good functional outcome reported in the literature using SCG as interpositional material supports its usage.

\section{Case Report}

A 15-year-old girl was referred to oral and maxillofacial surgery department for treatment of right TMJ ankylosis. Her right mandible become hypoplastic. she gave history of birth trauma. Clinical examination revealed an obviously hypoplastic mandible with a class II dental relationship. The mandibular midline was $3 \mathrm{~mm}$ to the right of the facial midline but the occlusal plane was not canted. Maximum intraincisal opening was $1 \mathrm{~mm}$ (Fig:1), there was no palpable movement over the right TMJ and only slight rotation on the left side. Prominent antigonial notch on the right side. Radiographic investigations confirm bony ankylosis of the right TMJ with elongation of the right coronoid processes.

Following treatment plan was carried out-

1. Surgery: Gap arthroplasty, Ipsilateral coronoidectomy, Harvesting of sternoclavicular joint graft and rigid internal fixation.
2. Physiotherapy: Aggressive use of continuous passive movement (CPM)

The surgery was done under general anesthesia with tracheotomy intubation. Submandibular and preauricular approach was used to reach the site of ankylotic mass. Right sided gap arthroplasty and coronoidectomy were done. Same sided sternoclavicular joint graft was selected for harvest and harvesting was done through infraclavicular transverse incision. Harvesting graft was replaced in the gap (Fig.2) and fixed to right ramus with 2 bicortical screws. Care was taken to maintain vertical height of ramus. Then lateral pteregoid muscle was sutured with sternoclavicular joint graft. Closing was done layer by layer. Immediately 2 days after surgery her mouth opening was $30 \mathrm{~mm}$, there was no posterior gaging and anterior open bite, all TMJ movement were slightly present.

In the follow up visit her mouth opening was increase (35 $\mathrm{mm})$ (Fig 3), occlusion is satisfactory, chin throat angle was decrease so improvement of facial esthetics, her chest x-ray reveled partially bone formation of harvested site of clavicle, patient satisfaction is very high.

\section{Discussion}

TMJ ankylosis is a distressing clinical condition causing complex problems as inability to open the mouth, chewing difficulties, speech impairment, facial asymmetry, airway compromise as well as a psychological stress to the patients. The main objectives of the treatment for Temporomandibular joint ankylosis are to increase mouth opening, restore joint function, initiation of mandibular growth, improve the patient's facial esthetic profile and prevent re-ankylosis. ${ }^{1}$ The various techniques for surgical correction of TMJ ankylosis are gap arthroplasty, interpositional arthroplasty and Joint reconstruction. ${ }^{3}$

In this case report unilateral ankylosis was considered for case selection. Unilateral TMJ ankylosis presents with, a short ramus, flat gonial angle, deviation of chin to affected side and hence there is a need to provide a graft with 


\section{Photographs}

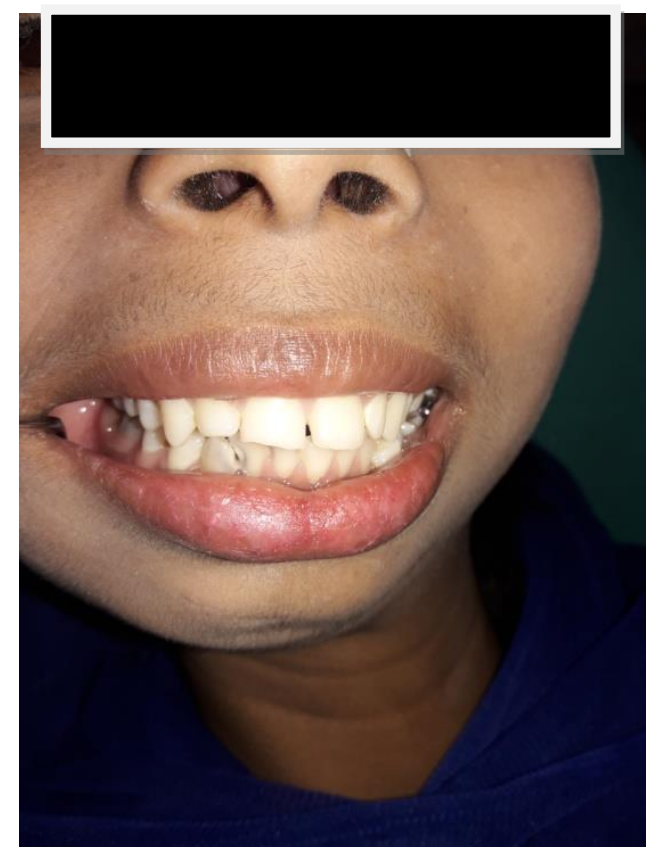

Fig.1: Preoperative photograph

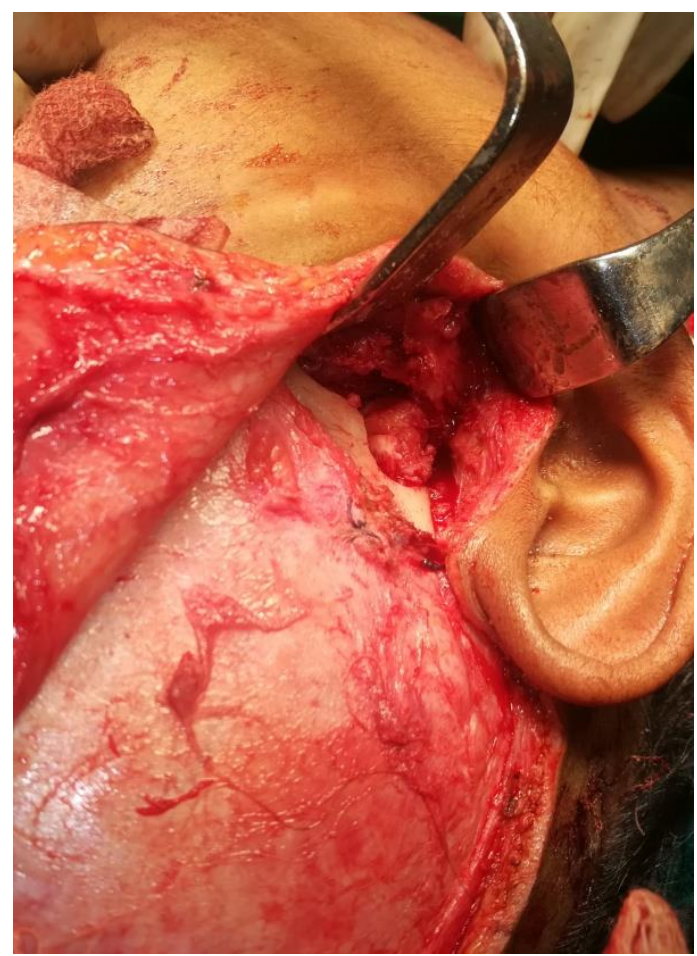

Fig. 2: Sternoclavicular joint was replaced in the gap

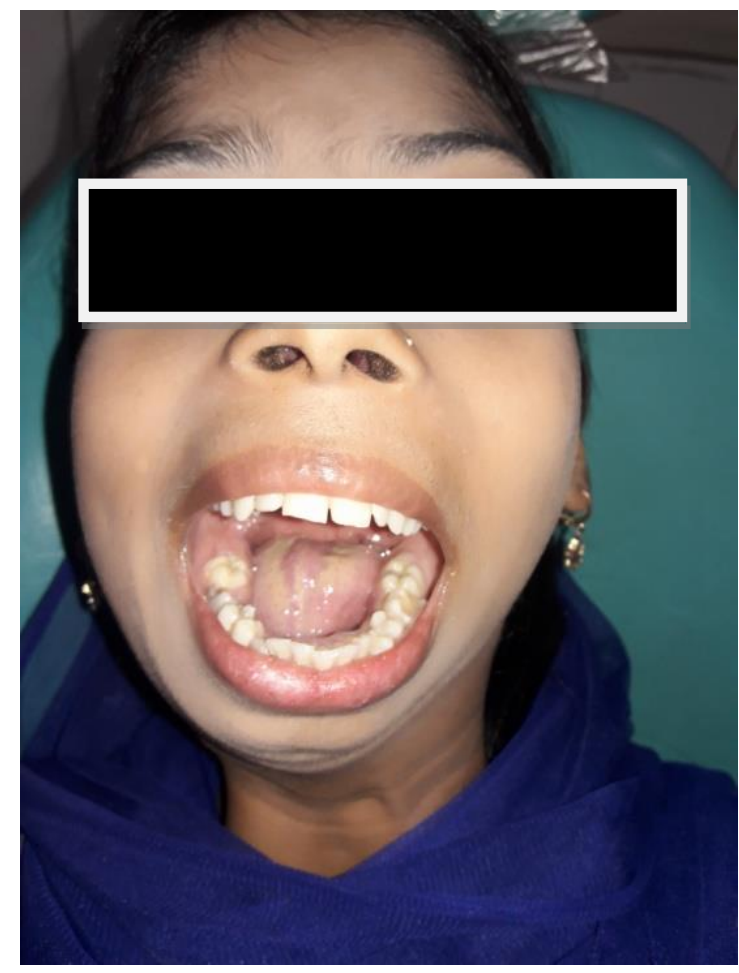

Fig. 3: Post-operative mouth opening (35 mm) in follow up visit 
growth capacities similar to normal condyle. Autogenous graft or alloplastic materials have been used by many surgeons to reconstruct the TMJ. The disadvantages of alloplastic material are wear and tear of the material, foreign body reactions, loosening of the implant or displacement, fracture, high cost, dystrophic bone formation, lack of growth potential in children. In spite of having drawbacks such as donor site morbidity, infection, failure of uptakes, autogenous grafts have been still widely used for TMJ reconstruction.

The various autogenous grafts used are costochondral. ${ }^{6,7,18}$ sternoclavicular 9,10,11,12,13 metatarsal, iliac crest, fibula, coronoid. The most widely used autogenous graft costochondral graft was first described by Harold Gilles 6 however the current technique was popularized by Poswillo ${ }^{8}$. Costochondral graft (CCG) is biologically compatible, easily workable adapts into the new environment, there is a potential for the donor site to grow or regenerate but CCG have many drawbacks that made us to search for an alternative. Sternoclavicular graft which resembles the TMJ morphologically and histologically could be a viable option. ${ }^{11}$ Snyder et al reported using first sternoclavicular whole joint graft consisting of manubrium, with capsule and a portion of clavicle on a 70 year old man with cylindroma ${ }^{14}$ Ried et al reported a free flap technique that included the clavicular head of pectoralis major and overlying skin to provide a vascularised free graft. ${ }^{9}$ Seimssen (1982) used clavicle pedicle on clavicular head of sterno mastoid muscle as a graft for TMJ reconstruction. ${ }^{10}$ Ellis and Carlson (1986) did a histological comparison of costochondral, sterno clavicular and TMJ during growth in Macaca Mullatta and found that the TMJ and SCJ are very similar morphologically throughout the growth period and that the clavicular head had layers of cartilage typical to the mandibular condyle, However the CCJ did not resemble the condyle but rather appeared similar to growth plate in a long bone epiphysis during growth and concluded that SCJ may be more suitable for mandibular condyle replacement. ${ }^{15}$ Sarnet and Laskin postulated that SCJ and TMJ are similar anatomically and physiologically. The SCJ articulation has a growth centre, interarticular fibro articular disc that simulates the disk of the TMJ.

In this case, there was a complete regeneration of clavicle minimizing donor site morbidity. The post operative complications were nil except for a mild scar in the donor site, which was acceptable. The postoperative mouth opening was highly satisfied with a maximum mouth opening of more than $35 \mathrm{~mm}$. The growth of the reconstructed TM joint was evaluated with orthopantomogram, \& CT scan. The complete regeneration of the SCG was also observed with chest $x$ ray. The reconstruction of TMJ with SCG has been proved to be successful in this reported case.

\section{Conclusion}

The outcome revealed that anatomic resemblance of SCG to TMJ and there was no donor site morbidity. The mouth opening was normal (more than $35 \mathrm{~mm}$ ) during the follow up period after the reconstruction of the TMJ with SCG. The patients were subjectively satisfied with the outcome. With an impressive benefit, the sternoclavicular graft could be a versatile and viable alternative to surgeons in reconstruction of TMJ and relatively simple to carry out with minimal complications and good results.

\section{References}

1. Peter wardbooth-oral and maxillofacial surgery-second edition

2. Kaban LB, Bouchard C, Troulis MJ. A protocol for management of temporomandibular joint ankylosis in children. J Oral Maxillofac Surg 2009;67:1966-78.

3. Alexander Katsnelson, Michael $R$. Markiewicz, David A. Keith, and Thomas B. Dodson, Operative Management of 
Temporomandibular Joint Ankylosis: A Systematic Review and Meta-Analysis. J Oral Maxillofac Surg 2012; 70:531- 536.

4. Singh V, Verma A, Kumar I, Bhagol A: Reconstruction of ankylosed temporomandibular joint. sternoclavicular grafting as an approach to management. Int $\mathrm{J}$ Oral Maxillofac Surg 2011;40:260-5.

5. Daniels S, Ellis 3rd E, Carlson DS. Histologic analysis of costochondral and sternoclavicular grafts in the TMJ of the juvenile monkey. $\mathrm{J}$ Oral Maxillofac Surg 1987;45:675-83.

6. Gillies H. Plastic Surgery of the Face. London: Oxford University Press; 1920.

7. Politis C, Fossion E, Bossuyt M:The use of costochondral grafts in arthroplasty of the temporomandibular joint. $\mathrm{J}$ Craniomaxillofac Surg 1987; 15: 345-354.

8. Poswillo DE: Biological reconstruction of the mandibular condyle. Br J Oral

Maxillofac Surg 1987;25:100-4.

9. Reid CD, Taylor GI, Waterhouse N. The clavicular head of pectoralis major

musculocutaneous free flap. $\mathrm{Br} \mathrm{J}$ Plast Surg 1986;39:57-65.

10. Siemssen SO: Temporomandibular arthroplasty by transfer of the Sternoclavicular joint on a muscle pedicle. Br J Plast Surg 1982; 35:225,

11. Singh V, Verma A, Kumar I, Bhagol A: Reconstruction of ankylosed temporomandibular joint. sternoclavicular grafting as an approach to management. Int $\mathrm{J}$ Oral MaxillofacSurg 2011;40:260-5.

12. Virendra Singh, Rahul Dhingra,and Amrish Bhagol:Prospective Analysis of Temporomandibular Joint Reconstruction in Ankylosis With Sternoclavicular Graft and Buccal Fat Pad Lining J Oral Maxillofac Surg $2012 ; 70: 997-1006$

13. Wolford LM, Cottrell DA, Henry C. Sternoclavicular grafts for temporomandibular joint reconstruction. J Oral Maxillofac Surg 1994;52:119-28

14. Snyder CC, Levine GA, Dingman DL. Trial of a sternoclavicular whole joint graft as a substitute for the temporomandibular joint. Plast Reconstr Surg 1971; 48:447-52.
15. Ellis III E, Carlson DS. Histologic comparison of the costochondral, sternoclavicular, and temporomandibular joints during growth in Macaca mulatta. J Oral MaxillofacSurg 1986;44:312-21. 